\title{
Whole cell imaging of Plasmodium falciparum blood stages
}

\author{
Eric Hanssen ${ }^{*}$, Christian Knoechel ${ }^{* *}$, Peter Carlton ${ }^{* * *}$, John Sedat ${ }^{* * *}$, Carolyn Larabell ${ }^{* * * * * * *}$, \\ Leann Tilley *, \\ *Department of Biochemistry and Center of Excellence for Coherent X-ray Science.La Trobe \\ University, Melbourne, VIC, 3086, Australia \\ ** National Center for X-ray Tomography, Lawrence Berkeley Laboratory, Berkeley, CA 94720, \\ USA \\ ${ }^{* * *}$ Department of Biochemistry and Biophysics, University of California, San Francisco, CA 94143, \\ USA \\ ${ }^{* * * *}$ Department of Anatomy, University of California San Francisco, San Francisco, CA 94143, USA
}

During intra-erythrocytic development, the human malaria parasite, Plasmodium falciparum, reorganizes its host's cell by exporting proteins beyond its own plasma membrane. It is faced with a complete lack of endogenous protein trafficking machinery within the host red blood cell, and therefore has to install its own apparatus. The Maurer's clefts (MC) are a crucial component of this protein sorting and trafficking machinery [1]. MC are parasite-derived membranous whose architecture is not well characterized, current model range from single isolated membranous structures linked by vesicular trafficking through to a highly interconnected network that in some models link the parasitophorous membrane to the erythrocyte surface.

We have conducted electron tomographic reconstruction in order to characterize new isolated structures in this trafficking route. But in order to describe the $\mathrm{MC}$ "network" we had to investigate means of imaging whole cells. While we have previously shown, using confocal microscopy, that the interconnection between $\mathrm{MC}$ is limited [2], structural illumination, serial section tomography (Fig 1), X-ray tomography and dual beam microscopy in conjunction with mutagenesis have led to new and unexpected insight in the MC architecture. [3]

\section{References}

[1] Lanzer M et al., Int J Parasitol 36 (2006) 23.

[2] Hanssen E et al.,Mol Microbiol 67(4) (2008) 703.

[3] This research was supported by Australian Research Council, the US Department of Energy, Office of Biological and Environmental Research (DE-AC02-05CH1 1231), the National Center for Research Resources of the National Institutes of Health (P41RR019664), the National Institutes of General Medicine of the National Institutes of Health (GM63948) and the U. S. Department of Energy, Office of Science. We thank A Awasthi, W-W Gu, D Parkinson and E Wilson for technical support. We thank M Ryan, La Trobe University, B Cooke, Monash University, and P Beck, for antibodies. 


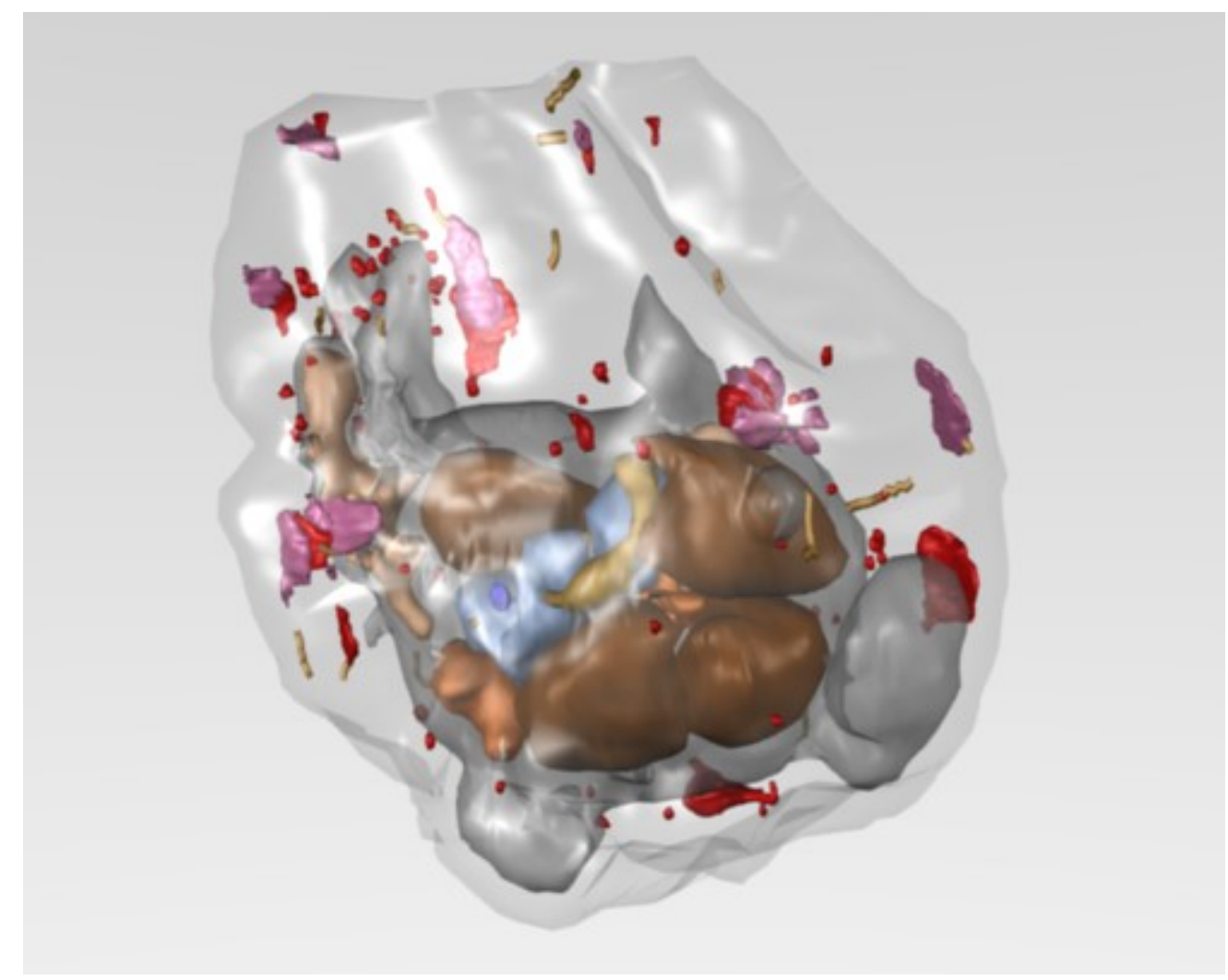

FIG. 1. segmentation model of whole cell tomography on a D10 parasite. Pink and Red, Maurer's clefts, transparent red blood cell; grey, TVN; brown, nucleus; blue, food vacuole; yellow, mitochondrion; orange, cytosotomes. 